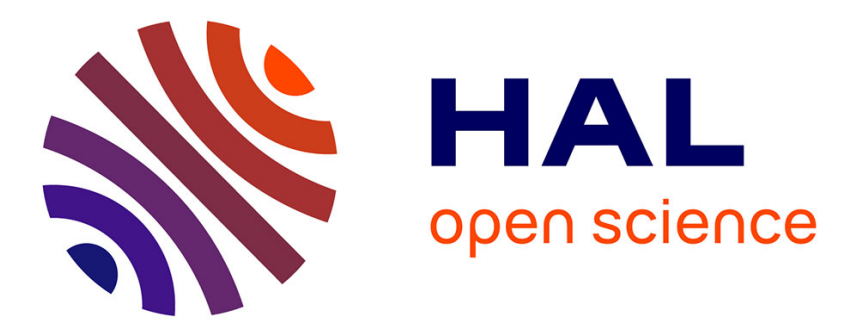

\title{
Intrinsic quantum confinement in formamidinium lead triiodide perovskite
}

Adam D Wright, George Volonakis, Juliane Borchert, Christopher L Davies, Feliciano Giustino, Michael B Johnston, Laura M. Herz

\section{- To cite this version:}

Adam D Wright, George Volonakis, Juliane Borchert, Christopher L Davies, Feliciano Giustino, et al.. Intrinsic quantum confinement in formamidinium lead triiodide perovskite. Nature Materials, 2020, 19 (11), pp.1201-1206. 10.1038/s41563-020-0774-9 . hal-02930358

\section{HAL Id: hal-02930358 https://hal.science/hal-02930358}

Submitted on 23 Nov 2020

HAL is a multi-disciplinary open access archive for the deposit and dissemination of scientific research documents, whether they are published or not. The documents may come from teaching and research institutions in France or abroad, or from public or private research centers.
L'archive ouverte pluridisciplinaire HAL, est destinée au dépôt et à la diffusion de documents scientifiques de niveau recherche, publiés ou non, émanant des établissements d'enseignement et de recherche français ou étrangers, des laboratoires publics ou privés. 


\title{
Intrinsic quantum confinement in formamidinium lead triiodide perovskite
}

Adam D. Wright ${ }^{1}$, George Volonakis ${ }^{2,3}$, Juliane Borchert ${ }^{1}$, Christopher L. Davies $^{1}$, Feliciano Giustino ${ }^{2,4,5}$, Michael B. Johnston ${ }^{1}$ and Laura M. Herz ${ }^{1 *}$

Email: laura.herz@physics.ox.ac.uk

${ }^{1}$ Department of Physics, University of Oxford, Clarendon Laboratory, Parks Road, Oxford OX1 3PU, United Kingdom

${ }^{2}$ Department of Materials, University of Oxford, Parks Road, Oxford OX1 3PH, United Kingdom

${ }^{3}$ Univ Rennes, ENSCR, INSA Rennes, CNRS, ISCR (Institut des Sciences Chimiques de Rennes), UMR 6226, Rennes F-35000, France

${ }^{4}$ Oden Institute for Computational Engineering and Sciences, The University of Texas at Austin, Austin, Texas 78712, USA

${ }^{5}$ Department of Physics, The University of Texas at Austin, Austin, Texas 78712, USA

\begin{abstract}
Understanding the electronic energy landscape in metal halide perovskites is essential for further improvements in their promising performance in thin-film photovoltaics. Here, we uncover the presence of above-bandgap oscillatory features in the absorption spectra of formamidinium lead triiodide thin films. We attribute these discrete features to intrinsically occurring quantum confinement effects, for which the related energies change with temperature according to the inverse square of the intrinsic lattice parameter, and with peak index in a quadratic fashion. By determining the threshold film thickness at which the amplitude of the peaks is appreciably decreased, and through ab initio simulations of the absorption features, we estimate the length scale of confinement to be $10-20 \mathrm{~nm}$. Altogether, such absorption peaks present a novel and intriguing quantum electronic phenomenon in a nominally bulk semiconductor, offering intrinsic nanoscale optoelectronic properties without necessitating cumbersome additional processing steps.
\end{abstract}

Metal halide perovskites are renowned for their spectacular performance as photovoltaic 
active layers [1, 2], with single-junction perovskite solar cells now achieving power conversion efficiencies (PCEs) of over 25\%[3]. This success stems from their excellent material properties, such as high charge-carrier mobilities, low exciton binding energies, broad absorption across the solar spectrum[1, 4], and facile fabrication routes [5]. While perovskite solar cells typically incorporate thin films of 3D (bulk) perovskite, nanostructures have also been engineered not only for solar cells, but also LEDs, lasers and photodetectors 6]. Whether nanocrystals (0D), nanowires (1D) or nanoplates (2D) are employed, the reduced dimensionality results in quantum confinement of charge carriers which yields dramatically different optoelectronic properties, including enhanced photoluminescence quantum yield[7] and lower thresholds for amplified spontaneous emission [8].

Although the creation of such perovskite nanostructures has clear advantages, it often relies on challenging top-down fabrication methods[6, 9]. It would therefore be highly advantageous if instead nanoscale domains were found to form intrinsically through selfassembly in the perovskite. Intrinsic domains have in fact already been observed [10, 11, 12, 13, 14] in films of methylammonium lead triiodide $\left(\mathrm{MAPbI}_{3}=\mathrm{CH}_{3} \mathrm{NH}_{3} \mathrm{PbI}_{3}\right)$. However, while these polar domains may be associated with electronic barriers [15, 16, 17], their reported widths range between 90 and $300 \mathrm{~nm}[11$, 12, 13], roughly an order of magnitude too large to result in appreciable quantum confinement effects [9, 6]. Intriguingly, there have been some recent suggestions that these domains are ferroelectric [13, 14, although others have instead considered them to arise from ferroelastic effects[12, 18]. In addition, the presence of mobile ions has been found to mimic ferroelectric signatures[12, making this a contentious topic.

Interestingly, formamidinium lead triiodide $\left(\mathrm{FAPbI}_{3}=\mathrm{CH}\left(\mathrm{NH}_{2}\right)_{2} \mathrm{PbI}_{3}\right)$ may be a far more promising candidate than $\mathrm{MAPbI}_{3}$ for displaying intrinsic quantum confinement. The proposed presence of twin domains bounded by the non-perovskite $\delta$-phase of $\mathrm{FAPbI}_{3}[19$, 20] could, for example, lead to the formation of intrinsic nanostructures. In addition, as detailed in Supplementary Note 13, the combination of the greater polar deformation and smaller dipole-dipole interaction energy attributable to the FA cation[21] gives reason[22] to expect any polar ferroelectric domains in $\mathrm{FAPbI}_{3}$ to be smaller than 
in $\mathrm{MAPbI}_{3}$ and hence engender more significant quantum confinement. Compared to $\mathrm{MAPbI}_{3}, \mathrm{FAPbI}_{3}$ is more resistant to heat stress 23] and has a narrower, more optimal band gap for solar cells[23, 24, 25]. It furthermore exhibits good charge-carrier mobility [26], long charge-carrier diffusion length [26] and impressive PCEs in solar cells [27]. Therefore, while $\mathrm{FAPbI}_{3}$ has been investigated to a far lesser extent than $\mathrm{MAPbI}_{3}$, we identify it as a promising candidate to display intrinsic quantum confinement.

In this work, we report the discovery of intrinsically-occurring nanostructures in $\mathrm{FAPbI}_{3}$, which exhibit quantum confinement effects manifested as an oscillatory absorption feature above the band gap. These features are present at room temperature but sharpen and become more apparent as the temperature is lowered towards $4 \mathrm{~K}$. We demonstrate that the energetic spacings and temperature-dependence of the peaks vary in a manner consistent with quantum confinement intrinsically associated with the lattice of the material. We suggest the origin of this confinement to be nanodomains with an extent of approximately $10-20 \mathrm{~nm}$. This interpretation is supported by correlating absorption spectra against $a b$ initio calculations based on the bandstructure of $\mathrm{FAPbI}_{3}$ in the presence of infinite barriers, and simulations for superlattices with moderate barrier heights. We further explore ferroelectricity/ferroelasticity and delta-phase twin boundaries as two possible causes of these domains. Altogether, such oscillatory absorption of $\mathrm{FAPbI}_{3}$ presents an intriguing intrinsic quantum electronic phenomenon in a highly promising semiconducting material.

Fig. 17 reveals oscillatory features in the absorption spectrum of $\mathrm{FAPbI}_{3}$ films, which are just discernible at room temperature but become much more prominent towards lower temperatures. $\mathrm{FAPbI}_{3}$ thin films were fabricated by dual-source vapour deposition, as detailed in Supplementary Note 1, which produces highly uniform and smooth thin films [28] exhibiting minimal scattering effects and sharp absorption onsets, as evident from Fig. 11a. Both reflection and transmission measurements (see inset) were taken from $4 \mathrm{~K}$ up to $295 \mathrm{~K}$ using a Fourier transform infrared (FTIR) spectrometer, and the absorption coefficient was obtained as detailed in Supplementary Note 3. We took great care to ensure that the observed oscillatory features did not result from optical interference (Fabry-Pérot 
oscillations), were not specific to the measurement technique, or conflicting with what is known to date of $\mathrm{FAPbI}_{3}$ thin-film absorption (see Supplementary Note 3). We further proved that they were specific to the semiconducting perovskite phases of $\mathrm{FAPbI}_{3}$ thin films alone (they were absent in its yellow $\delta$-phase) and did not appear in a $\mathrm{MAPbI}_{3}$ thin film measured under identical conditions (Supplementary Note 3).

Fig. 19 demonstrates that for $\mathrm{FAPbI}_{3}$, the typically smoothly rising absorption onset of a semiconductor is modulated with additional peak features. To allow for better visualisation and quantification of the oscillatory peaks, we therefore decouple them from the underlying absorption spectrum, albeit only phenomenologically. As shown in the inset to Fig. 1 b, we fitted a spline baseline to the above-bandgap absorption data using the procedure detailed in Supplementary Note 4. Subtracting this baseline from the absorption spectrum yielded solely the peak features, whose temperature dependence is shown in Fig. 1 $\mathrm{b}$ for a 297 nm-thick $\mathrm{FAPbI}_{3}$ film, and for other film thicknesses in Supplementary Figure 10. In isolation from the overall absorption spectra, the peaks can be clearly seen to increase in both amplitude and energy spacing with increasing energy, and to reduce in amplitude with temperature.

We further find that the energies of the peak maxima display subtle shifts with temperature (Fig. 1b). However, such shifts may also derive from modifications in the band gap energy $E_{\mathrm{g}}$ of the semiconductor, rather than changes in quantization energy with temperature. To decouple such effects we therefore determine $E_{\mathrm{g}}$ by modelling the onsets of the measured $\mathrm{FAPbI}_{3}$ absorption spectra with fits based on Elliott's theory (Supplementary Note 4 \& Supplementary Figures 9 and 11), which describes the absorption spectra of semiconductors as arising from both bound excitons and electron-hole continuum states [29], and has been successfully applied to metal halide perovskites [25, 30, 31]. The resultant values of $E_{\mathrm{g}}$ (Fig. $2 \mathrm{a}$ ) and the exciton binding energy (Supplementary Figure 12) for the six films ranging in thickness from $35 \mathrm{~nm}$ to $840 \mathrm{~nm}$, are consistent with previous reports [25, 24, 32, 33] and with PL measurements (Supplementary Note 5). We note that $E_{\mathrm{g}}$ decreases slightly with increasing film thickness, which may result from the increased lattice strain present in thinner films [9]. 

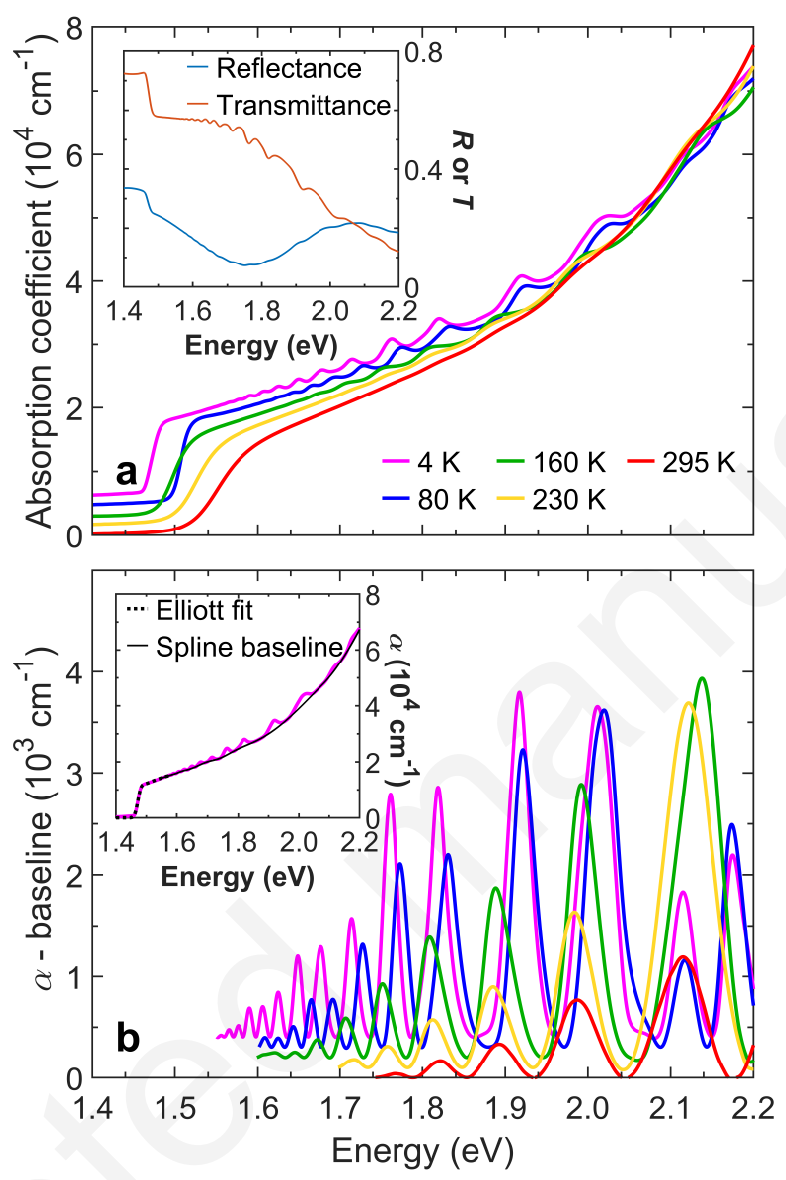

Figure 1: Temperature-dependent absorption coefficient and peak features. a) Absorption spectra and b) absorption peaks for a 297-nm thick $\mathrm{FAPbI}_{3}$ film, measured by FTIR spectroscopy at roughly $70 \mathrm{~K}$ temperature intervals, from $4 \mathrm{~K}$ (magenta) to $295 \mathrm{~K}$ (red). For clarity, spectra and peaks at successively decreasing temperatures are offset vertically by $1.5 \times 10^{3} \mathrm{~cm}^{-1}$ and $1 \times 10^{2} \mathrm{~cm}^{-1}$ respectively. The upper inset depicts the reflectance $(\mathrm{R}$, blue) and transmittance $(\mathrm{T}$, red) of the film at $4 \mathrm{~K}$. The lower inset shows the absorption coefficient at $4 \mathrm{~K}$ with its absorption onset fitted by a function based on Elliott's theory (dotted black line) and the oscillatory region fitted with a spline baseline fit (solid black line), which is subtracted from the absorption coefficient to give the peaks shown in (b). 

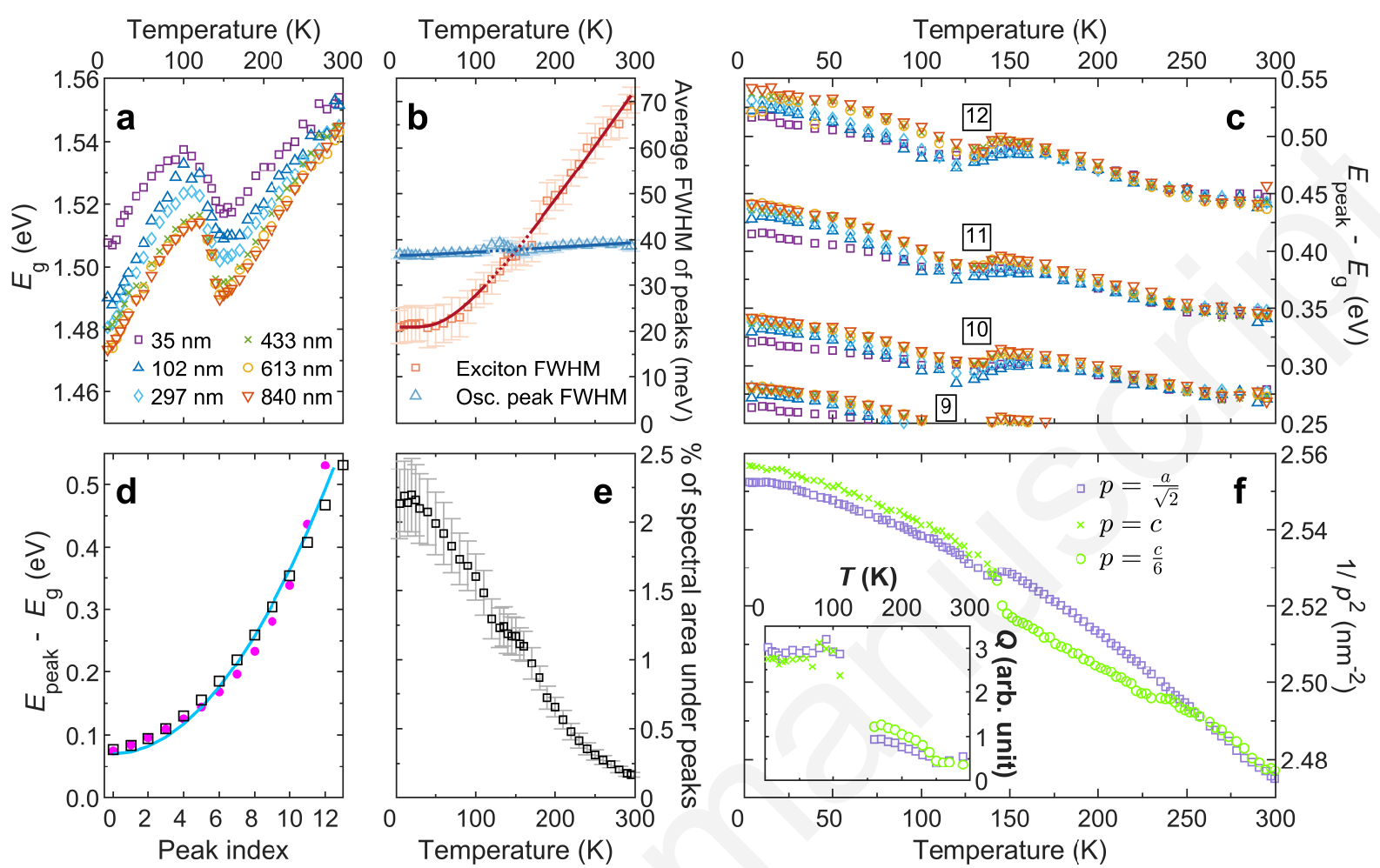

Figure 2: Temperature-dependence of optoelectronic and lattice properties of $\mathbf{F A P b I}_{3}$. a) Band gap $\left(E_{\mathrm{g}}\right)$ extracted from Elliott fits to the absorption spectra for films of six thicknesses indicated by the legend. b) Linewidth (FWHM) of the excitonic peak extracted from the Elliott fits (red squares) and the most prominent peaks (blue triangles), averaged across the six film thicknesses. The dark solid lines are fits which account for Fröhlich coupling with LO phonons, excluding temperatures near the phase transition (dotted lines). Error bars show the standard error. c) Energies of the peak maxima $\left(E_{\text {peak }}\right)$ relative to the band gap $E_{\mathrm{g}}$. Boxed numbers show the peak indices. d) Variation of $E_{\text {peak }}-E_{\mathrm{g}}$ (magenta dots) at $4 \mathrm{~K}$ in the $297-\mathrm{nm}$ thick film, as a function of the peak index. The solid blue line is a fit based on a quadratic function while the black squares represent band energies derived from a Krönig-Penney potential (See Supplementary Note 7 ). The offset of the lowest-energy peaks from $E_{\mathrm{g}}$ may be attributed to a slightly higher band gap within the $2 \mathrm{D}$ nanoplate, shifted by $\approx 70 \mathrm{meV}$ due to lattice strain within the nanostructure [23, 9]. e) Integral (area) under the main peaks (black squares) as a percentage of the total area under the spectrum, averaged across the six thicknesses. Error bars show the standard error. f) Temperature dependence of $1 / p^{2}$ for reduced lattice parameters $p$, adapted with permission from ref. [34] (Copyright 2018 American Chemical Society). The inset shows $Q$, which is given by the ratio of the temperature gradients in $E_{\text {peak }}-E_{\mathrm{g}}$ to those in $1 / p^{2}$, for the 297 -nm thick film (see Supplementary Note 8). 
From the positions of the peak energies $\left(E_{\text {peak }}\right)$ relative to the band gap $E_{\mathrm{g}}$ (Fig. 2r) we are able to demonstrate through multiple pieces of evidence that quantum confinement effects cause the observed absorption features. Firstly, comparison between the temperature dependencies of $E_{\mathrm{g}}$ (Fig. 2a) and $E_{\mathrm{g}}-E_{\text {peak }}$ (Fig. 22) clearly proves that the peaks are influenced by temperature independently of the band gap and mostly also of film thickness (see also Supplementary Note 6 and Supplementary Figure 14). In fact, the peaks generally shift in the opposite direction from $E_{\mathrm{g}}$ with temperature, with a small interruption by the transition from the $\gamma$-phase to the $\beta$-phase near $140 \mathrm{~K}$. These trends can be fully understood if they are considered to arise from changes in the lattice constant of $\mathrm{FAPbI}_{3}$, which lead to a concomitant reduction of the intrinsic domains or substructure from which the quantum confinement derives. To examine such correlations, we considered the temperature dependence of the reduced lattice parameters [35] $(p=a / \sqrt{2}, c$ for the $\beta$-phase, $p=a / \sqrt{2}, c / 6$ for the $\gamma$-phase) of $\mathrm{FAPbI}_{3}$, taken from a previous neutron diffraction report [34]. As discussed in more detail below, if the peaks observed in the absorption spectra arise from the presence of intrinsic electronic barriers, for example, in the form of a quasi-infinite potential well, or a superlattice, then the confinement energy $E_{\text {peak }}-E_{\mathrm{g}}$ will depend on the inverse square of the well width or superlattice period (see Supplementary Note 7). Since these lengths scale with the lattice constant $p$, any changes in the associated confinement energies must be proportional to $1 / p^{2}$. Fig. 2 indeed reveals that $1 / p^{2}$ has a very similar dependence on temperature to $E_{\text {peak }}-E_{\mathrm{g}}$, for both sets of reduced lattice parameters on either side of the $\beta$ - $\gamma$ phase transition. To indicate this similarity more quantitatively, the inset to Fig. 2f shows the ratio $Q$ of the temperature gradients of $1 / p^{2}$ and $E_{\text {peak }}-E_{\mathrm{g}}$ (for peak series 12 of the 297-nm thick film, see Supplementary Note 8 for details). Away from the phase transition, $Q$ appears relatively temperature-independent, suggesting that the changes in confinement energy $E_{\text {peak }}-E_{\mathrm{g}}$ indeed mostly derive from modifications in the extent of the intrinsic domains in $\mathrm{FAPbI}_{3}$ that give rise to the quantum confinement.

Secondly, our assignment of these peaks to electronic confinement is also supported by the quadratic relationship between peak index $n$ and the confinement energy $E_{\text {peak }}-E_{\mathrm{g}}$, 
displayed in Fig. 2d. To illustrate this point, we examine two confinement potential scenarios, represented by either the simple 'particle in a box' model of an infinite quantum well, or a superlattice comprising a periodic arrangement of quantum wells separated by semi-permeable barriers (see Supplementary Note 7 for full details). Both models accurately predict the above-gap energy to scale with $n^{2}$, as observed, because the associated $k$-vector scales with the inverse of the quantum well width, or period, respectively. However, as we show in Supplementary Notes $7 \& 10$, for the case of the superlattice represented by a Krönig-Penney potential, more moderate (finite) potential barriers can still give rise to peak features at energies exceeding $E_{\mathrm{g}}$ by more than the potential height. We suggest such superlattices may derive from clusters containing nanostructures of similar extent, with our calculated band energies expected for a superlattice (plotted as black squares in Fig. 2d) agreeing well with our observed peak positions. These arguments therefore confirm that peaks may arise from electronic barriers present in the material in the form of quantum wells or coupled superlattices.

As a third point, we note that the peaks are broadened in a fundamentally very different manner from the bulk states associated with the excitonic peak near the absorption onset. Fig. 2b compares the temperature-dependent broadening of the peaks (plotted in blue) with the broadening of the bulk excitonic states (plotted in red), showing distinctly different magnitudes and trends. Through approximating such temperature-dependent broadening to arise from electronic coupling to optical phonons (see Supplementary Note 6) we determine an optical phonon energy $E_{\mathrm{LO}}=19 \mathrm{meV}$ and coupling strength $\gamma_{\mathrm{LO}}=57$ meV, for the broadening associated with the excitonic absorption onset, broadly consistent with previous reports for this material[36, 24]. In contrast, the corresponding fit to the linewidths of the additional peak features (solid blue line in Fig. $2 \mathrm{~b}$ ) yield substantially lower values of only $0.7 \mathrm{meV}$ and $6 \mathrm{meV}$, respectively, indicating a considerably different origin of the broadening. As an explanation, we note that a reduction in coupling strength to optical phonons is symptomatic of semiconductors with reduced dimensionality [37, 38, again pointing towards quantum confinement as the cause of the peaks.

If quantum confinement is intrinsic to semiconducting $\mathrm{FAPbI}_{3}$, one way to explore its 
origin is to consider the effective length scale of the structure to which charge carriers are confined. We tackle this challenge in a two-fold approach, first by examining the dependence of the peaks on film thickness, and second through ab initio calculations aimed at reproducing the observed features from calculations based on the $\mathrm{FAPbI}_{3}$ bandstructure under confinement. Our first experimental approach reveals that the positions of the peaks have little dependence on the $\mathrm{FAPbI}_{3}$ film thickness in the range between $35 \mathrm{~nm}$ and 840 nm (see Supplementary Figure 10 and derived data in Fig. 22). This observation proves that the film surfaces themselves do not provide the boundaries of electronic confinement, as we would otherwise expect the energy of confinement to scale roughly inversely with the square of the film thickness. Therefore, a different, intrinsic length scale of quantum confinement may be involved, likely to be significantly smaller than the film thickness. To explore the magnitude of this intrinsic scale, we reduced the nominal film thickness further, to below $35 \mathrm{~nm}$ and to the point where the peaks had appreciably weakened in amplitude. We note that down to a thickness of $35 \mathrm{~nm}$, the strength of the absorption peaks observed had also been relatively independent of film thickness (see Supplementary Figure 10). However, as indicated in Fig. 3 a, once the film thickness is further lowered to $10 \mathrm{~nm}$, the amplitude of the peaks at $4 \mathrm{~K}$ has decreased by at least a factor four compared with the 35-nm thick film sample, and peaks are no longer visible at room temperature (Supplementary Figure 22). The tenuous presence of absorption peaks in the 10-nm thick film suggests that its thickness is close to the minimum at which this phenomenon can occur, and is hence similar to the length scale of confinement, with local ordering disrupted for film thicknesses that undercut this intrinsic domain size.

We further examine the length scale of confinement through density functional theory (DFT) [39, 40, 41] calculations that simulate quantum confinement effects on the absorption spectra of $\mathrm{FAPbI}_{3}$, here for the simple case of infinite potential barriers in all three dimensions. Full details for these calculations can be found in Supplementary Note 9. Fig. 3b shows the resultant absorption coefficient spectra, calculated using confinement lengths $\left(L_{\mathrm{DFT}}\right)$ ranging from $3 \mathrm{~nm}$ to $17 \mathrm{~nm}$. As expected, these calculated spectra exhibit peaks corresponding to the energy transitions between quantised valence and conduction 
band states which become more closely spaced with increasing $L_{\mathrm{DFT}}$. For comparison, we also display the experimentally measured absorption spectrum for the 297-nm thick film at $4 \mathrm{~K}$ (black line), which indicates that the best correspondence between the peak features in the measured and calculated spectra occurs for $L_{\mathrm{DFT}}=13 \mathrm{~nm}$ or $17 \mathrm{~nm}$. We therefore note that taking into account both experimental variation of film thickness, and $a b$ initio calculations, indicates that quantum confinement occurs on a length scale of 10-20 nm, which is feasible given our 10-15 nm estimate in Supplementary Note 14 of the excitonic Bohr radius in $\mathrm{FAPbI}_{3}$.

We note at this point though that we cannot definitively ascertain the number of dimensions the electronic system is confined in, as our baseline removal procedure may remove any underlying, continuous density of states that may also be associated with the quantum confinement. While electronic confinement by infinite potential wells in all three directions (as in our first-principles approach - see Fig. 3b) yields peak-like absorption features, one-dimensional confinement gives rise to additional continuum states associated with free motion in the other two dimensions (see Supplementary Notes 4 \& 10). As Figure 3r indicates, a one-dimensional superlattice displays absorption features modulating an otherwise relatively smooth underlying density of states (see Supplementary Note 10 for full calculations based on a model using a Krönig-Penney potential). While we thus cannot determine the dimensionality of confinement, or the fraction of the material that exhibits confinement, we find these to be consistent across different film thicknesses, likely because such dual-source vapour deposition has previously been found to result in highly uniform morphology 42$]$ independent of film thickness. We further note that the sharpness of the peak features modulating the $\mathrm{FAPbI}_{3}$ absorption spectra indicates the disorder attributable to any variation in the length scales of confinement to be relatively low.

Having uncovered the presence of quantum confinement in $\mathrm{FAPbI}_{3}$, we need to identify the origin of such potential wells. We propose two possible causes of intrinsic confinement in $\mathrm{FAPbI}_{3}$ : the presence of $\delta$-phase barriers, and the formation of nanoscale ferroelectric or -elastic domains. Our discussion accounts for the various trends observed, including the 

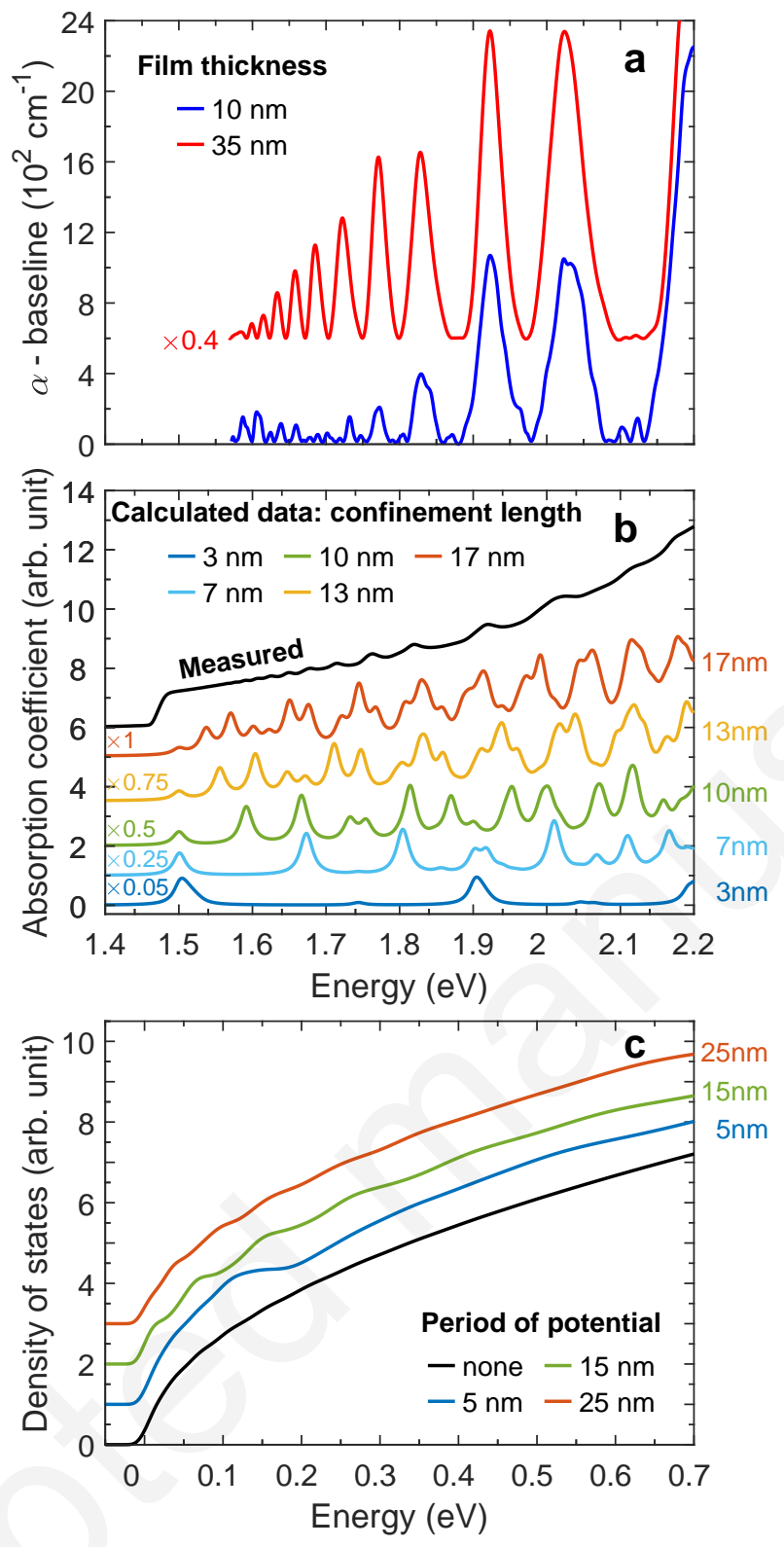

Figure 3: Estimating the lengthscale of structures inducing confinement. a) Measured absorption peaks of $10-\mathrm{nm}$ (blue) and 35-nm thick (red, offset vertically by $6 \times 10^{2} \mathrm{~cm}^{-1}$ and scaled vertically by a factor of 0.4 ) films of $\mathrm{FAPbI}_{3}$ at $4 \mathrm{~K}$. b) Measured and calculated absorption coefficient of $\mathrm{FAPbI}_{3}$. The calculated spectra are vertically offset from each other and scaled vertically by the factors indicated in the corresponding colors. The measured spectrum (black line), taken at $4 \mathrm{~K}$ for the $297-\mathrm{nm}$ thick film, uses a different vertical scale. The calculated spectra are based on a simple particle-in-a-box system with confinement in 3 dimensions, taking account of the actual bandstructure of $\mathrm{FAPbI}_{3}$ through first-principles calculations. A spectral broadening of $10 \mathrm{meV}$ is used for the calculated spectra, which are simulated at $0 \mathrm{~K}$ (see Supplementary Note 9). c) Calculated density of states (DOS) for a one-dimensional superlattice. Calculations are based on a model using a Krönig-Penney potential, assuming a square-like periodic wave potential, with wall width $b$ here set to one-tenth of the period $a+b$ (see Supplementary Note 10 for full details). Here, the DOS is shown for a fixed potential amplitude of $250 \mathrm{meV}$, but with variations in superlattice period $a+b$. The black line shows the free-electron case (with no periodic potential) representing a square-root function. 
clear weakening of the features with increasing temperature (Figure 2e). Moreover, the mild (70 meV) energetic offset of the first peaks from the bandgap (See Supplementary Note 7 and quadratic fit in Figure 2d) could indicate that lattice strain is prominent in the confinement regions [23, 9]. Furthermore, the successful performance of a device based on a similar film, shown in Supplementary Note 2 to achieve a short-circuit current density of $80 \%$ of the theoretical maximum, suggests a lack of impermeable barriers to charge motion, and may be compatible with the presence of an intrinsic electronic superlattice structure.

We first examine the possibility that small inclusions of non-perovskite $\delta$-phase could act as potential barriers within an otherwise semiconducting perovskite $\mathrm{FAPbI}_{3}$. The $\delta$-phase of $\mathrm{FAPbI}_{3}$ is yellow and so has a significantly wider bandgap than the perovskite phases, making it in principle suitable as a potential barrier material. In support of this argument, we note that $\mathrm{FAPbI}_{3}$ films that were originally in the perovskite $\alpha$-phase at room temperature have been found (using neutron diffraction) in some cases to contain $\delta$-phase upon cooling [43], although not in other measurements 34]. The absence 44] of an equivalent $\delta$-phase in $\mathrm{MAPbI}_{3}$ would further explain why we find similar peaks to be absent in its absorption spectra. Although DFT calculations have predicted that $\{111\}$ twin boundaries (which structurally correspond to the $\delta$-phase) in $\mathrm{FAPbI}_{3}$ are associated with an electronic barrier height of less than $100 \mathrm{meV}$ [20], a periodic superlattice with such low potential barriers corresponding to $\delta$-phase twin boundaries could still cause absorption peaks at higher energies, as shown in Supplementary Notes $7 \& 10$. We find prominent features to arise from such structures even for thin barriers of less than $10 \%$ of the width of the potential wells between them, which could still be compatible with the apparent absence of a visible $\delta$-phase peak in the X-ray diffraction patterns of our $\mathrm{FAPbI}_{3}$ films within their detection limit (see Supplementary Figure 1). In addition, we note that while the absorption peaks seem to disappear above a temperature of $\sim 380 \mathrm{~K}$ (Supplementary Figure 23), which is near the temperature at which the $\delta$-phase transitions to the $\alpha$-phase[34, these features can re-appear when the sample is cooled again. While $\alpha$-phase is usually meta-stable at room temperature for an extended period [34], it is 
possible that strained regions may respond with the spontaneous formation of $\delta$-phase superlattices upon cooling.

An alternative possibility is that the absorption peaks arise from quantum confinement within ferroelectric or -elastic domains in $\mathrm{FAPbI}_{3}$. As detailed in the introduction, such domains had not to date been observed in $\mathrm{FAPbI}_{3}$, but have been examined in more detail in $\mathrm{MAPbI}_{3}$, for which they have been proposed to be[12, 13] between 90-300 nm wide. Based on comparisons of dielectric properties for the two materials (see Supplementary Note 13) we would estimate polar domains in $\mathrm{FAPbI}_{3}$ to be approximately twenty times smaller, in good agreement with the length scales of $10-20 \mathrm{~nm}$ on which we find chargecarriers to be quantum confined. Ferroelectric domain walls in $\mathrm{PbTiO}_{3}\left[45\right.$, and $\mathrm{BiFeO}_{3}[46$ have been calculated to present potential steps of up to $180 \mathrm{meV}$, which is again a feasible height for a periodic superlattice potential to cause absorption features such as those we observe. However, ferroelectricity has been extensively debated for $\mathrm{FAPbI}_{3}$ 's cousin $\mathrm{MAPbI}_{3}$, for which some authors 47, 12] consider this phenomenon to be impossible at room-temperature. Our data are not necessarily in contention with this claim, given that we definitely observe a temperature-dependent decline in the amplitude of the peaks (Fig. 2 2 ) which is similar in shape to that observed for the spontaneous polarisation of a ferroelectric material with a second-order phase transition[48]. In addition, the reappearance of the peaks when cooling down from $380 \mathrm{~K}$ mirrors the spontaneous reappearance of polarisation in ferroelectrics below their Curie temperature 13 . Indeed, such behaviour is also a feature of ferroelasticity, the previously mentioned phenomenon which has been proposed as an alternative cause of the domains observed in $\mathrm{MAPbI}_{3}[12$, 18]. Thus we believe that quantum confinement may alternatively arise from either ferroelectric or ferroelastic domains in $\mathrm{FAPbI}_{3}$.

In summary, we have discovered novel peak features in the absorption spectra of $\mathrm{FAPbI}_{3}$ arising from discrete optical transitions of electrons between quantum confined states and potentially caused by formation of ferroelastic or polar ferroelectric domains, or $\delta$-phase inclusions. Our analysis is supported by relative peak energies with respect to the band edge scaling with temperature according the inverse square of the intrinsic lat- 
tice parameter, and with peak index in a quadratic fashion. By observing a threshold film thickness of $10 \mathrm{~nm}$ at which the amplitude of peaks is appreciably decreased, and through $a b$ initio simulations of the absorption features, we estimate the length scale of quantum confinement to be 10-20 nm. These observations represent an exciting discovery because they offer the highly sought-after goal of electronic quantum confinement without the requirement of extensive top-down nano-processing steps, while maintaining efficient flow of electrical current. Such nanostructures offer the narrowed-down densities of states required to enhance radiative emission, lower thresholds for amplified spontaneous emission, and facilitate electrical injection lasing.

\section{Data availability}

The datasets generated during and/or analysed during the current study are available in the Oxford University Research Archive repository at https://doi.org/10.5287/ bodleian:Z52M67emQ.

\section{Supplementary Information}

Supplementary Information accompanies this paper at ...

\section{Acknowledgements}

This work was supported by the Engineering and Physical Sciences Research Council, the EPSRC Center for Doctoral Training in New and Sustainable Photovoltaics, the Chaire de Recherche Rennes Metropole project and the Robert A. Welch Foundation under award F-1990-20190330. 


\section{Author Contributions}

A.D.W. performed the FTIR experiments, data analysis and participated in the experimental planning. G.V. carried out the first-principles calculations. J.B. prepared the samples. C.L.D. provided support with the FTIR experiments and data analysis. The project was conceived, planned and supervised by F.G., M.B.J. and L.M.H.. A.D.W. wrote the first version of the manuscript and all authors contributed to the discussion and preparation of the final version of the article.

\section{Competing interests}

The authors declare no competing interests.

\section{References}

[1] Johnston, M. B. \& Herz, L. M. Hybrid Perovskites for photovoltaics: charge-carrier recombination, diffusion, and radiative efficiencies. Acc. Chem. Res. 49, 146-154 (2016).

[2] Kojima, A., Teshima, K., Shirai, Y.\& Miyasaka, T. Organometal halide perovskites as visible-light sensitizers for photovoltaic cells. J. Am. Chem. Soc. 131, 6050-6051 (2009).

[3] NREL. NREL Research Cell Record Efficiency Chart (2019).

[4] Herz, L. M. Charge-carrier dynamics in organic-inorganic metal halide perovskites. Annu. Rev. Phys. Chem. 67, 65-89 (2016).

[5] Stoumpos, C. C. \& Kanatzidis, M. G. Halide perovskites: poor man's highperformance semiconductors. Adv. Mater. 28, 5778-5793 (2016).

[6] Fu, Y. et al. Metal halide perovskite nanostructures for optoelectronic applications and the study of physical properties. Nat. Rev. Mater. 4, 169-188 (2019). 
[7] Polavarapu, L., Nickel, B., Feldmann, J. \& Urban, A. S. Advances in quantumconfined perovskite nanocrystals for optoelectronics. Adv. Energy Mater. 7, 1-9 (2017).

[8] Li, M. et al. Amplified spontaneous emission based on 2D Ruddlesden - Popper perovskites. Adv. Funct. Mater. 28, 1707006 (2018).

[9] Parrott, E. S. et al. Growth modes and quantum confinement in ultrathin vapourdeposited $\mathrm{MAPbI}_{3}$ films. Nanoscale 11, 14276-14284 (2019).

[10] Hermes, I. M. et al. Ferroelastic fingerprints in methylammonium lead iodide perovskite. J. Phys. Chem. C 120, 5724-5731 (2016).

[11] Rothmann, M. U. et al. Direct observation of intrinsic twin domains in tetragonal $\mathrm{CH}_{3} \mathrm{NH}_{3} \mathrm{PbI}_{3}$. Nat. Commun. 8, 6-13 (2017).

[12] Wilson, J. N., Frost, J. M., Wallace, S. K. \& Walsh, A. Dielectric and ferroic properties of metal halide perovskites. APL Mater. 7, 010901 (2019).

[13] Röhm, H. et al. Ferroelectric properties of perovskite thin films and their implications for solar energy conversion. Adv. Mater. 31, 1806661 (2019).

[14] Röhm, H., Leonhard, T., Hoffmann, M. J. \& Colsmann, A. Ferroelectric poling of methylammonium lead iodide thin films. Adv. Funct. Mater. 30, 1908657 (2020).

[15] Liu, S. et al. Ferroelectric domain wall induced band gap reduction and charge separation in organometal halide perovskites. J. Phys. Chem. Lett. 6, 693-699 (2015).

[16] Montero-Alejo, A. L., Menéndez-Proupin, E., Palacios, P., Wahnón, P. \& Conesa, J. C. Ferroelectric domains may lead to two-dimensional confinement of holes, but not of electrons, in $\mathrm{CH}_{3} \mathrm{NH}_{3} \mathrm{PbI}_{3}$ perovskite. J. Phys. Chem. C 121, 26698-26705 (2017).

[17] Pecchia, A., Gentilini, D., Rossi, D., Auf der Maur, M. \& Di Carlo, A. Role of ferroelectric nanodomains in the transport properties of perovskite solar cells. Nano Lett. 16, 988-992 (2016). 
[18] Gómez, A., Wang, Q., Goñi, A. R., Campoy-Quiles, M. \& Abate, A. Reply to the "Comment on the publication 'Ferroelectricity-free lead halide perovskites' by Gomez et al." by Colsmann et al. Energy Environ. Sci. 13, 1892-1895 (2020).

[19] Weller, M. T., Weber, O. J., Frost, J. M. \& Walsh, A. Cubic perovskite structure of black formamidinium lead iodide, $\alpha$ - $\left[\mathrm{HC}\left(\mathrm{NH}_{2}\right)_{2}\right] \mathrm{PbI}_{3}$, at 298 K. J. Phys. Chem. Lett. 6, 3209-3212 (2015).

[20] McKenna, K. P. Electronic properties of $\{111\}$ twin boundaries in a mixed-ion lead halide perovskite solar absorber. ACS Energy Letters 3, 2663-2668 (2018).

[21] Frost, J. J. M. J. et al. Atomistic origins of high-performance in hybrid halide perovskite solar cells. Nano Lett. 14, 2584-2590 (2014).

[22] Lines, M. E. \& Glass, A. M. Principles and Applications of Ferroelectrics and Related Materials (Oxford University Press, Oxford, UK, 1977).

[23] Eperon, G. E. et al. Formamidinium lead trihalide: a broadly tunable perovskite for efficient planar heterojunction solar cells. Energy Environ. Sci. 7, 982-988 (2014).

[24] Wright, A. D. et al. Electron-phonon coupling in hybrid lead halide perovskites. Nat. Commun. 7, 11755 (2016).

[25] Davies, C. L. et al. Impact of the organic cation on the optoelectronic properties of formamidinium lead triiodide. J. Phys. Chem. Lett. 9, 4502-4511 (2018).

[26] Rehman, W. et al. Charge-carrier dynamics and mobilities in formamidinium lead mixed-halide perovskites. Adv. Mater. 27, 7938-7944 (2015).

[27] Li, Y. et al. Formamidinium-based lead halide perovskites: structure, properties, and fabrication methodologies. Small Methods 2, 1700387 (2018).

[28] Borchert, J. et al. Large-area, highly uniform evaporated formamidinium lead triiodide thin films for solar cells. ACS Energy Lett. 2, 2799-2804 (2017). 
[29] Elliott, R. J. Intensity of optical absorption by excitions. Phys. Rev. 108, 1384-1389 (1957).

[30] Davies, C. et al. Bimolecular recombination in methylammonium lead triiodide perovskite is an inverse absorption process. Nat. Commun. 9, 293 (2018).

[31] Sestu, N. et al. Absorption F-sum rule for the exciton binding energy in methylammonium lead halide perovskites. J. Phys. Chem. Lett. 6, 4566-4572 (2015).

[32] Francisco-López, A. et al. Phase diagram of methylammonium/formamidinium lead iodide perovskite solid solutions from temperature-dependent photoluminescence and raman spectroscopies. J. Phys. Chem. C 124, 3448-3458 (2020).

[33] Wright, A. D. et al. Band-tail recombination in hybrid lead iodide perovskite. Adv. Funct. Mater. 27, 1700860 (2017).

[34] Weber, O. J. et al. Phase behavior and polymorphism of formamidinium lead iodide. Chem. Mater. 30, 3768-3778 (2018).

[35] Brivio, F. et al. Lattice dynamics and vibrational spectra of the orthorhombic, tetragonal and cubic phases of methylammonium lead iodide. Phys. Rev. B 92, $144308(2015)$.

[36] Fang, H.-H. et al. Photoexcitation dynamics in solution-processed formamidinium lead iodide perovskite thin films for solar cell applications. Light Sci. Appl 5, e16056 (2016).

[37] Bockelmann, U. \& Bastard, G. Phonon scattering and energy relaxation in two-, one-, and zero-dimensional electron gases. Phys. Rev. B 42, 8947-8951 (1990).

[38] Alivisatos, A. P., Harris, T. D., Carroll, P. J., Steigerwald, M. L. \& Brus, L. E. Electron-vibration coupling in semiconductor clusters studied by resonance Raman spectroscopy. J. Chem. Phys. 90, 3463-3468 (1989).

[39] Marini, A., Hogan, C., Grüning, M. \& Varsano, D. yambo: An ab initio tool for excited state calculations. Comp. Phys. Commun. 180, 1392-1403 (2009). 
[40] Giannozzi, P. et al. QUANTUM ESPRESSO: a modular and open-source software project for quantum simulations of materials. J. Phys.: Condens. Matter. 21, 395502 (2009).

[41] Perdew, J. P., Burke, K. \& Ernzerhof, M. Generalized gradient approximation made simple. Phys. Rev. Lett. 77, 3865-3868 (1996).

[42] Crothers, T. W. et al. Photon re-absorption masks intrinsic bimolecular chargecarrier recombination in $\mathrm{CH}_{3} \mathrm{NH}_{3} \mathrm{PbI}_{3}$ rerovskite. Nano Lett. 17, 5782-5789 (2017).

[43] Chen, T. et al. Entropy-driven structural transition and kinetic trapping in formamidinium lead iodide perovskite. Sci. Adv. 2, e1601650 (2016).

[44] Stoumpos, C., Malliakas, C. \& Kanatzidis, M. Semiconducting tin and lead iodide perovskites with organic cations: phase transitions, high mobilities, and near-infrared photoluminescent properties. Inorg. Chem. 52, 9019-9038 (2013).

[45] Meyer, B. \& Vanderbilt, D. Ab initio study of ferroelectric domain walls in $\mathrm{PbTiO}_{3}$. Phys. Rev. B 65, 104111 (2002).

[46] Seidel, J. et al. Conduction at domain walls in oxide multiferroics. Nat. Mater. 8, 229-234 (2009).

[47] Gómez, A., Wang, Q., Goñi, A. R., Campoy-Quiles, M. \& Abate, A. Ferroelectricityfree lead halide perovskites. Energy Environ. Sci. 12, 2537-2547 (2019).

[48] Martin, L. W. \& Rappe, A. M. Thin-film ferroelectric materials and their applications. Nat. Rev. Mater. 2, 16087 (2016).

[49] Chen, T. et al. Origin of long lifetime of band-edge charge carriers in organic-inorganic lead iodide perovskites. PNAS 114, 7519-7524 (2017).

[50] Wright, A. D. et al. Dataset for ' Intrinsic quantum confinement in formamidinium lead triiodide perovskite'. Oxford University Research Archive 10.5287/bodleian:Z52M67emQ , (2020). 


\section{Methods}

\section{Sample preparation}

Formamidinium lead triiodide thin films were grown on z-cut quartz substrates using a dual-source thermal evaporation system (Kurt J. Lesker) as reported previously [28, 25]. The precursors were FAI purchased from Greatcell Solar Materials and $\mathrm{PbI}_{2}$ from Alfa Aesar. These precursors were placed in separate alumina crucibles and heated until they evaporated. The samples were mounted on a rotating substrate holder to enable uniform deposition. The deposited films were annealed at $170{ }^{\circ} \mathrm{C}$ for $1 \mathrm{~min}$ to ensure they were in the desired perovskite phase of $\mathrm{FAPbI}_{3}$.

\section{Device fabrication}

A solar cell based on an evaporated $\mathrm{FAPbI}_{3}$ film was fabricated using a standard architecture. The substrate was fluoride-doped tin oxide (FTO) coated glass, onto which the fullerene $\mathrm{C}_{60}$ from Acros Organics was evaporated at $350{ }^{\circ} \mathrm{C}$. The perovskite layer was co-evaporated onto this as described above. Next the hole extraction material $2,2^{\prime}, 7,7^{\prime}-$ Tetrakis-(N,N-di-4-methoxyphenylamino)-9,9'-spirobifluorene (Spiro-OMeTAD) from Lumtec was spin-coated on top. Spiro-OMeTAD was doped with Lithium bis(trifluoromethanesulfonyl)imide (LiTFSI) and 4-tert-Butylpyridine (tBP) and the solution spin coated at $2000 \mathrm{rpm}$ for $45 \mathrm{~s}$. Finally, 100nm of silver were evaporated to contact the solar cells. The size of the evaporated metal contacts was $0.0919 \mathrm{~cm}^{2}$.

\section{X-ray diffraction}

X-ray diffraction (XRD) patterns of $\mathrm{FAPbI}_{3}$ films were measured in air using a Panalytical X'pert powder diffractometer with a copper X-ray source. 


\section{Current-voltage measurements}

Current-voltage $(\mathrm{J}-\mathrm{V})$ measurements were carried out on the solar cell device (based on an evaporated $\mathrm{FAPbI}_{3}$ film) under illumination with an ABET class AAB sun 2000 simulator. The scan speed was $0.38 \mathrm{~V} \mathrm{~s}^{-1}$.

\section{Reflection-transmission measurements}

To measure the temperature-dependent reflectance and transmittance of $\mathrm{FAPbI}_{3}$ films, a Bruker Vertex 80v Fourier transform infrared (FTIR) spectrometer was used, config-

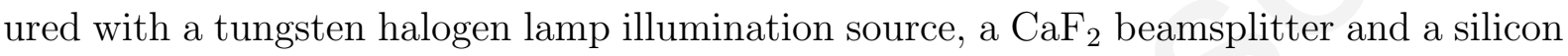
detector. The samples were mounted in a gas-exchange helium cryostat (Oxford Instruments, OptistatCF2) and heated over temperatures from 4 to $295 \mathrm{~K}$ in increments of between 5 and $10 \mathrm{~K}$.

\section{Photoluminesence measurements}

To measure the temperature-dependent photoluminescence of $\mathrm{FAPbI}_{3}$ films, the sample was photoexcited by a $398 \mathrm{~nm}$ picosecond pulsed diode laser (PicoHarp, LDH-D-C-405M). The resultant photoluminescence was collected and coupled into a grating spectrometer (Princeton Instruments, SP-2558), which directed the spectrally dispersed PL onto a silicon iCCD (PI-MAX4, Princeton Instruments). The sample was mounted under vacuum $\left(\mathrm{P}<10^{-6} \mathrm{mbar}\right)$ in a cold-finger liquid helium cryostat (Oxford Instruments, MicrostatHe) and heated over temperatures from 4 to $295 \mathrm{~K}$ in increments of between 3 and $6 \mathrm{~K}$

\section{First-principles calculations}

The electronic band structure of $\mathrm{FAPbI}_{3}$ at $0 \mathrm{~K}$ was simulated using a simplified, abstract cubic lattice structure which lacks the FA cation. This method avoided the complexities of the FA cation orientation, while still ensuring that the band gap energy and band dispersion were consistent with experimentally measured values [49]. Next, the effect of electronic confinement was introduced to this band structure by generating a discrete 
$k$-point grid of the wavevectors allowed for a particle confined by infinite barriers in all three dimensions within a confinement length $L$. The absorption coefficient arising from such confined electronic states was then computed as resulting from transitions between the conduction band and valence band states lying on the $k$-point grid of our simulated band structure.

The datasets generated during and/or analysed during the current study are available in the Oxford University Research Archive repository [50]. 